University of Wollongong

Research Online

Faculty of Business - Papers (Archive)

Faculty of Business and Law

$1-1-2018$

Fractal attractors in economic growth models with random pollution externalities

Davide La Torre

University of Milan

Simone Marsiglio

University of Wollongong, simonem@uow.edu.au

Fabio Privileggi

University of Turin

Follow this and additional works at: https://ro.uow.edu.au/buspapers

Part of the Business Commons

Research Online is the open access institutional repository for the University of Wollongong. For further information contact the UOW Library: research-pubs@uow.edu.au 


\title{
Fractal attractors in economic growth models with random pollution externalities
}

\author{
Abstract \\ We analyze a discrete time two-sector economic growth model where the production technologies in the \\ final and human capital sectors are affected by random shocks both directly (via productivity and factor \\ shares) and indirectly (via a pollution externality). We determine the optimal dynamics in the \\ decentralized economy and show how these dynamics can be described in terms of a two-dimensional \\ affine iterated function system with probability. This allows us to identify a suitable parameter \\ configuration capable of generating exactly the classical Barnsley's fern as the attractor of the log- \\ linearized optimal dynamical system. \\ Disciplines \\ Business \\ Publication Details \\ La Torre, D., Marsiglio, S. \& Privileggi, F. (2018). Fractal attractors in economic growth models with \\ random pollution externalities. Chaos, 28 (5), 055916-1-055916-12.
}

This journal article is available at Research Online: https://ro.uow.edu.au/buspapers/1448 


\section{Fractal attractors in economic growth models with random pollution externalities}

Davide La Torre, Simone Marsiglio, and Fabio Privileggi

Citation: Chaos 28, 055916 (2018); doi: 10.1063/1.5023782

View online: https://doi.org/10.1063/1.5023782

View Table of Contents: http://aip.scitation.org/toc/cha/28/5

Published by the American Institute of Physics

\section{Articles you may be interested in}

A financial market model with two discontinuities: Bifurcation structures in the chaotic domain Chaos: An Interdisciplinary Journal of Nonlinear Science 28, 055908 (2018); 10.1063/1.5024382

Time-varying economic dominance in financial markets: A bistable dynamics approach

Chaos: An Interdisciplinary Journal of Nonlinear Science 28, 055903 (2018); 10.1063/1.5021141

Introduction to the focus issue "nonlinear economic dynamics"

Chaos: An Interdisciplinary Journal of Nonlinear Science 28, 055801 (2018); 10.1063/1.5039304

2D discontinuous piecewise linear map: Emergence of fashion cycles

Chaos: An Interdisciplinary Journal of Nonlinear Science 28, 055917 (2018); 10.1063/1.5018588

Narrative dynamics in social groups: A discrete choice model

Chaos: An Interdisciplinary Journal of Nonlinear Science 28, 055915 (2018); 10.1063/1.5025177

Time delays, population, and economic development

Chaos: An Interdisciplinary Journal of Nonlinear Science 28, 055909 (2018); 10.1063/1.5024397

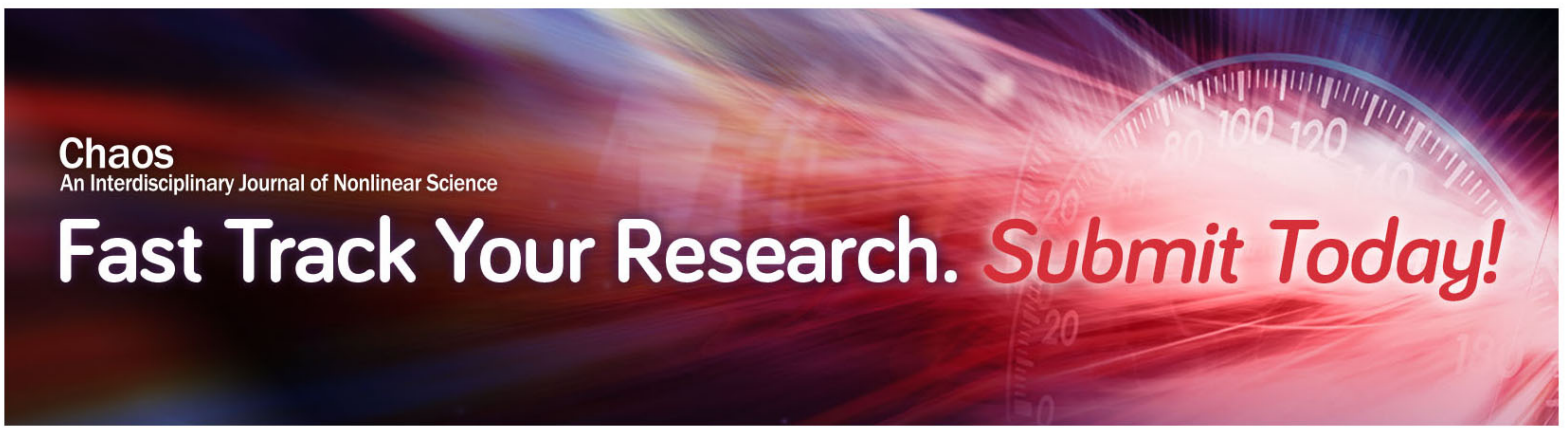




\title{
Fractal attractors in economic growth models with random pollution externalities
}

\author{
Davide La Torre, ${ }^{1, a)}$ Simone Marsiglio, ${ }^{2, b)}$ and Fabio Privileggi ${ }^{3, c)}$ \\ ${ }^{1}$ Dubai Business School, University of Dubai, Dubai, UAE, and Department of Economics, Management, \\ and Quantitative Methods, University of Milan, Milano 20122, Italy \\ ${ }^{2}$ School of Accounting, Economics and Finance, University of Wollongong, Wollongong, NSW 2522, Australia \\ ${ }^{3}$ Department of Economics and Statistics “de Martiis," University of Turin, Torino 10153, Italy
}

(Received 28 January 2018; accepted 23 March 2018; published online 31 May 2018)

\begin{abstract}
We analyze a discrete time two-sector economic growth model where the production technologies in the final and human capital sectors are affected by random shocks both directly (via productivity and factor shares) and indirectly (via a pollution externality). We determine the optimal dynamics in the decentralized economy and show how these dynamics can be described in terms of a twodimensional affine iterated function system with probability. This allows us to identify a suitable parameter configuration capable of generating exactly the classical Barnsley's fern as the attractor of the log-linearized optimal dynamical system. Published by AIP Publishing.

https://doi.org/10.1063/1.5023782
\end{abstract}

In this paper, we build a two-sector growth model that, thanks to pollution externalities randomly affecting output and human capital production, allows for a suitable parameterization that generates the Classical Barnsley's fern as the unique attractor of the economy.

\section{INTRODUCTION}

Over the last two decades, a large and growing number of studies have tried to characterize the eventual fractal nature of the steady state in economic models. Indeed, since the pioneering work by Montrucchio and Privileggi (1999) it has been well known that traditional macroeconomic models may give rise to random dynamics possibly converging to invariant measures supported on fractal sets. Several works tried to identify the conditions under which this might be the case by borrowing from the iterated function system (IFS) literature (Hutchinson, 1981; Vrscay, 1991; and Barnsley, 1993). Most of these works analyze discrete time stochastic economic growth models with logarithmic utility and CobbDouglas production, either in a one-sector or two-sector framework, showing that through appropriate logtransformations their optimal dynamics can be converted into an affine iterated function system converging to a singular measure supported on some fractal set; in the case of unidimensional iterated function systems such an attractor can be the Cantor set (Montrucchio and Privileggi, 1999; Mitra et al., 2003; Mitra and Privileggi, 2004; 2006; 2009; Marsiglio, 2012; Privileggi and Marsiglio, 2013; and La Torre et al., 2015), while in the case of two-dimensional iterated function systems it can be either the Sierpinski gasket or distorted-copies of the Barnsley's fern (La Torre et al., 2011; 2015; 2018). With the exception of La Torre et al. (2018)

\footnotetext{
${ }^{\text {a)} E l e c t r o n i c ~ a d d r e s s e s: ~ d l a t o r r e @ u d . a c . a e ~ a n d ~ d a v i d e . l a t o r r e @ u n i m i . i t . ~}$

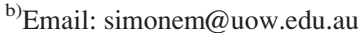

${ }^{c)}$ Email: fabio.privileggi@unito.it
}

who considered also shocks affecting factor shares, in all these works randomness affects economic activities through the productivity channel, following the Brock and Mirman (1972) tradition. Some of these works also identify specific parameter configurations under which the invariant probability measure turns out to be either singular or absolutely continuous (Mitra et al., 2003 and La Torre et al., 2015; 2018).

We contribute to this stochastic growth and fractal attractor literature by analyzing an economy in which economic production is affected by random shocks both directly (via productivity and factor shares) and indirectly (via a pollution externality). Specifically, we build on the model by La Torre et al. (2018) in which such direct effects have already been accounted for in order to allow for pollution to be an additional and indirect source of randomness. To the best of our knowledge, none of the extant works has ever considered how the presence of externalities complicates aggregate macroeconomic dynamics and what this may imply for the attractor of the associated iterated function system. However, accounting for the existence of such a pollution externality, as widely discussed in the environmental economics literature, is important to better characterize potential economic outcomes. Several papers document and discuss the extent to which the economy and the environment are mutually related (IPCC, 2007 and Nordhaus, 2013): on the one hand, economic activities generate pollution which is the primary determinant of environmental problems; on the other hand, environmental degradation precludes pollution absorption which in turn critically determines economic capabilities. Moreover, due to the large degree of uncertainty associated with environmental phenomena, very little is known with precision about such a bilateral economyenvironment relation, which is most likely to be random (Soretz, 2003 and Marsiglio and La Torre, 2016). In order to take these issues into account in the most intuitive way, we focus on the optimal dynamics in the decentralized economy, where the externality is not internalized yet but it fully affects 
the evolution of both control and state variables; such a setting is the most appropriate to give rise to a simple but realistic characterization of real world dynamics. We show how the optimal dynamics can be described in terms of a twodimensional affine iterated function system with probability, whose coefficients can eventually take on negative values, differently from all extant papers. Such a peculiarity of our framework, due to the presence of the pollution externality, allows us to identify a suitable parameter configuration capable of generating exactly the classical Barnsley's fern as the attractor of the log-linearized optimal dynamical system. To the best of our knowledge, no other macroeconomic model has ever been able to give rise to the Barnsley's fern [since the coefficients in their iterated function system can only take on positive values, La Torre et al. (2018) model is able to generate only distorted copies of the fern, which ultimately do not even remotely resemble a fern] for some specific_-but fully admissible-parameter configuration.

The rest of the paper is organized as follows: In Sec. II, we briefly recall some basic results from the mathematical theory on the iterated function system, which will help us discuss our following results. In Sec. III, we formally introduce our model which consists of a two-sector economic growth model subject to random shocks affecting economic productive activities both directly and indirectly, thanks to the role played by pollution. We also derive the optimal rule for the control variables and the optimal dynamics of the state variables-physical and human capital - in the decentralized economy in which the pollution externality is not internalized. In Sec. IV, we introduce a log-transformation which allows us to recast the nonlinear optimal dynamical system in terms of a twodimensional affine iterated function system, allowing us to borrow from the mathematical literature to discuss its eventual convergence to an invariant measure supported on some fractal set. In Sec. V, we present two specific model's parameterizations: the first one allows us to obtain exactly the classical Barnsley's fern as the attractor of the log-linearized optimal dynamics; the second one focuses on a more realistic scenario giving rise to an attractor very different from a fern. In Sec. VI, we conclude and present directions for future research, while the Appendix contains a brief sketch of the proof yielding the optimal dynamics of the model presented in Sec. III.

\section{PRELIMINARIES ON ITERATED FUNCTION SYSTEMS}

Let $(X, d)$ denote a compact metric space. An $N$-map iterated function system (IFS) on $X, \mathbf{w}=\left\{w_{1}, \ldots, w_{N}\right\}$, consists of $N$ contraction mappings on $X$, i.e., $w_{i}: X \rightarrow X$, $i=1, \ldots, N$, with contraction factors $c_{i} \in[0,1)$ (see Barnsley, 1993; Hutchinson, 1981; Barnsley et al., 1986; and Kunze et al., 2012). Associated with an $N$-map IFS, one can define a set-valued mapping $\hat{\mathbf{w}}$ on the space $\mathcal{H}([a, b])$ of nonempty compact subsets of $X$ as follows:

$$
\hat{\mathbf{w}}(S):=\bigcup_{i=1}^{N} w_{i}(S), \quad S \in \mathcal{H}([a, b]) .
$$

The following two results state a convergence property of an $N$-map IFS towards its attractor. More properties and results can be found in the study by Barnsley (1993), Hutchinson (1981), and Kunze et al. (2012).

Theorem 1 (Hutchinson, 1981). For $A, B \in \mathcal{H}(X)$

$$
h(\hat{\mathbf{w}}(A), \hat{\mathbf{w}}(B)) \leq \operatorname{ch}(A, B) \quad \text { where } c=\max _{1 \leq i \leq N} c_{i}<1
$$

and $h$ denotes the Hausdorff metric on $\mathcal{H}(X)$.

Corollary 1 (Hutchinson, 1981). There exists a unique set $A \in \mathcal{H}([a, b])$, the attractor of the IFS $\boldsymbol{w}$, such that

$$
A=\hat{\mathbf{w}}(A)=\bigcup_{i=1}^{N} w_{i}(A) .
$$

Moreover, for any $B \in \mathcal{H}([a, b]), \quad h\left(A, \hat{\mathbf{w}}^{n}(B)\right) \rightarrow 0$ as $n \rightarrow \infty$.

An N-map Iterated Function System with (constant) Probabilities (IFSP) $(\mathbf{w}, \mathbf{p})$ is an $N$-map IFS w with associated probabilities $\mathbf{p}=\left\{p_{1}, \ldots, p_{N}\right\}, \sum_{i=1}^{N} p_{i}=1$.

Let $\mathcal{M}(X)$ denote the set of probability measures on (Borel subsets of) $X$ and $d_{M K}$ the Monge-Kantorovich distance on this space: For $\mu, \nu \in \mathcal{M}(X)$, with the MongeKantorovich metric

$$
d_{M K}(\mu, \nu)=\sup _{f \in L i p_{1}(X)}\left[\int f \mathrm{~d} \mu-\int f \mathrm{~d} \nu\right]
$$

where $\operatorname{Lip}_{1}(X)=\{f: X \rightarrow \mathbb{R}|| f(x)-f(y) \mid \leq d(x, y)\}$. The metric space $\left(\mathcal{M}(X), d_{M K}\right)$ is complete (Barnsley, 1993; Hutchinson, 1981; and Kunze et al., 2012).

The Markov operator associated with an $N$-map IFSP is a mapping $M: \mathcal{M} \rightarrow \mathcal{M}$, defined as follows: For any $\mu \in \mathcal{M}(X)$, and for any measurable set $S \subset X$, define a measure $\nu=M \mu$ as

$$
\nu(S)=(M \mu)(S)=\sum_{i=1}^{N} p_{i} \mu\left(w_{i}^{-1}(S)\right) .
$$

The following results show that the Markov operator has a unique invariant measure $\bar{\nu}$ and it is globally attracting.

Theorem 2 (Hutchinson, 1981). For $\mu, \nu \in \mathcal{M}(X)$,

$$
d_{M K}(M \mu, M \nu) \leq c d_{M K}(\mu, \nu) .
$$

Corollary 2 (Hutchinson, 1981). There exists a unique measure $\bar{\nu} \in \mathcal{M}(X)$, the invariant measure of the IFSP $(\mathbf{w}, \mathbf{p})$, such that

$$
\bar{\mu}(S)=(M \bar{\mu})(S)=\sum_{i=1}^{N} p_{i} \bar{\mu}\left(w_{i}^{-1}(S)\right) .
$$

Moreover, for any $\nu \in \mathcal{M}(X), d_{M K}\left(\bar{\mu}, M^{n} \nu\right) \rightarrow 0$ as $n \rightarrow \infty$.

Theorem 3 (Hutchinson, 1981). The support of the invariant measure $\bar{\mu}$ of an $N$-map $\operatorname{IFSP}(\mathbf{w}, \mathbf{p})$ is the attractor A of the IFS w, i.e.,

$$
\operatorname{supp} \bar{\mu}=A .
$$

In order to determine the attractor of an IFSP, the following random dynamical system known as Chaos Game 
might be implemented: Starting from $x_{0} \in X$, let us determine $x_{t+1}=w_{\sigma}\left(x_{t}\right)$ where $\sigma$ is chosen in the interval $1 \ldots N$ with probabilities $p_{1}, \ldots, p_{N}$. It can be proved (see Kunze et al., 2012, for more details) that the orbit of this random dynamical system is dense in the attractor $A$.

\section{THE MODEL}

We analyze a discrete time two-sector economic growth model where the production technologies in the final and human capital sectors are affected by random shocks both directly (via productivity and factor shares) and indirectly (via pollution). While a large literature has considered the implications of random shocks for macroeconomic dynamics by analyzing the direct channel (Brock and Mirman, 1972; Montrucchio and Privileggi, 1999; Mitra et al., 2003; and La Torre et al., 2015; 2018), more limited is the number of works analyzing the indirect channel, and, in particular, the effects of pollution on aggregate macroeconomic dynamics (Privileggi and Marsiglio, 2013 and Marsiglio and La Torre, 2016). However, it is now well known that economic activities and environmental outcomes are mutually related, thus taking into account the fact that the existence of some economic-environmental feedback is essential to understand macroeconomic dynamics. Several studies discuss that pollution is a by-product of economic activities and how pollution impacts both aggregate economic activities and health (IPCC, 2007 and Nordhaus, 2013). We take these issues into account by (i) allowing output to be the driver of pollution and (ii) allowing pollution to affect both the productivity of the final output and that of human capital (meant as a broad form of capital encompassing not only education but health as well; Barro and Sala-i-Martin, 2004).

We analyze a standard two-sector optimal growth model under uncertainty, as discussed in the study by La Torre et al. (2011), extended to account for such mutual economicenvironmental feedback. Specifically, the representative household maximizes its lifetime utility subject to the evolution of physical and human capital. Lifetime utility is the infinite discounted ( $\beta>0$ is the discount factor) sum of instantaneous utilities, and the utility function is assumed to be logarithmic in consumption. At each time $t$, the household chooses its level of consumption, $c_{t}$, and which share of its human capital, $u_{t}$, to devote to the production of the final consumption good, which is produced according to a Cobb-Douglas technology combining physical, $k_{t}$, and human, $h_{t}$, capital. Also new human capital is produced according to a Cobb-Douglas technology, which however employs only human capital (Lucas, 1988 and Rebelo, 1991). As in the study by La Torre et al. (2018), the production technologies of the final good and new human capital are directly affected by exogenous shocks which take both a multiplicative form through coefficients $z_{t}$ and $\eta_{t}$, respectively, and an exponential form affecting the factor shares in both production functions; therefore, output is given by $y_{t}=z_{t} A_{t} k_{t}^{\alpha_{t}}\left(u_{t} h_{t}\right)^{\gamma_{t}}$, where $\alpha_{t}$ and $\gamma_{t}$ denote the random physical and human capital shares of income, respectively, while human capital is given by $h_{t+1}=\eta_{t} B_{t}\left[\left(1-u_{t}\right) h_{t}\right]^{\phi_{t}}$, with $\phi_{t}$ denoting the random human capital share of human capital. In this formulation, $A_{t}$ and $B_{t}$ denote the pollution-induced productivity levels in the final and human capital sectors, respectively.
Specifically, we assume that $A_{t}=P_{t}^{\nu_{t}}$ and $B_{t}=P_{t}^{\mu_{t}}$, where $P_{t}$ denotes pollution which is a by-product of macroeconomic activities and $\nu_{t}, \mu_{t} \in \mathbb{R}$ are random parameters; since these parameters can take on real values, this accounts for the fact that pollution may have positive or negative effects on the production of the final output and/or human capital. As in the study by Marsiglio et al. (2016), economic activities generate pollution according to $P_{t}=k_{t}^{\chi_{t}} h_{t}^{\omega_{t}}$ in order to represent that the production inputs are characterized by different pollution-intensities; such intensities are set by parameters $\chi_{t}$ and $\omega_{t}$, respectively, which, like $\nu_{t}$ and $\mu_{t}$, can take on real values and are random. This implies that $A_{t}=k_{t}^{\nu_{t} \chi_{t}} h_{t}^{\nu_{t} \omega_{t}}$ and $B_{t}=k_{t}^{\mu_{2} \chi_{t}} h_{t}^{\mu_{t} \omega_{t}}$, suggesting that randomness through the pollution channels affects indirectly the production technologies of the final good and human capital.

The whole $\left(z_{t}, \eta_{t}, \alpha_{t}, \gamma_{t}, \phi_{t}, \nu_{t}, \mu_{t}, \chi_{t}, \omega_{t}\right) \in \mathbb{R}^{9}$ is a random vector which is independent and identically distributed, and can take on $m$ values, i.e., at each time $t\left(z_{t}, \eta_{t}, \alpha_{t}, \gamma_{t}\right.$, $\left.\phi_{t}, \nu_{t}, \mu_{t}, \chi_{t}, \omega_{t}\right) \in\left\{\left(z_{i}, \eta_{i}, \alpha_{i}, \gamma_{i}, \phi_{i}, \nu_{i}, \mu_{i}, \chi_{i}, \omega_{i}\right)\right\}_{i=1}^{m}$. While shocks $z_{t}, \eta_{t}$ enter multiplicatively the two Cobb-Douglas production functions and $\alpha_{t}, \gamma_{t}, \phi_{t}$ represent shocks on the factor shares, $\nu_{t}, \mu_{t}, \chi_{t}, \omega_{t}$ are random externalities affecting final production through two channels: 1) $\nu_{t}, \mu_{t}$ determine how pollution modifies final production and 2) $\chi_{t}, \omega_{t}$ determine how much pollution is generated by the current levels of physical and human capital, $k_{t}$ and $h_{t}$, employed in the production of the composite good. As far as the first five shocks are concerned, we shall assume that $z_{t}, \eta_{t}>0$, $0<\alpha_{i}, \gamma_{i}, \phi_{i}<1$ and $\alpha_{i}+\gamma_{i} \leq 1$ for all $i=1, \ldots, m$. We do not impose any restriction on parameters $\nu_{i}, \mu_{i}, \chi_{i}, \omega_{i}$, but the realization of the shock determines whether physical or human capital is the relatively greener production input (according to how $\chi_{t}$ and $\omega_{t}$ compare). Each vector realization, $\left(z_{i}, \eta_{i}, \alpha_{i}, \gamma_{i}, \phi_{i}, \nu_{i}, \mu_{i}, \chi_{i}, \omega_{i}\right)$, occurs with (constant) probability $p_{i}$, with $p_{i} \in(0,1), i=1, \ldots, m$, and $\sum_{i=1}^{m} p_{i}=1$.

The optimization problem of the representative household can be summarized as follows:

$$
\begin{gathered}
V\left(k_{0}, h_{0}, z_{0}, \eta_{0}, \alpha_{0}, \gamma_{0}, \phi_{0}, \nu_{0}, \mu_{0}, \chi_{0}, \omega_{0}\right) \\
=\max _{\left\{c_{t}, u_{t}\right\}} \sum_{t=0}^{\infty} \beta^{t} \mathbb{E}_{0} \ln c_{t}, \\
\text { s.t. }\left\{\begin{array}{l}
k_{t+1}=z_{t} A_{t} k_{t}^{\alpha_{t}}\left(u_{t} h_{t}\right)^{\gamma_{t}}-c_{t} \\
h_{t+1}=\eta_{t} B_{t}\left[\left(1-u_{t}\right) h_{t}\right]^{\phi_{t}} \\
k_{0}>0, h_{0}>0,\left(z_{0}, \eta_{0}, \alpha_{0}, \gamma_{0}, \phi_{0}, \nu_{0}, \mu_{0}, \chi_{0}, \omega_{0}\right) \text { are given. }
\end{array}\right.
\end{gathered}
$$

As in the study by Benhabib and Perli (1994), in the competitive (decentralized) solution the representative households take $A_{t}$ and $B_{t}$ as given, meaning that, because of the pollution externality, this solution will differ from the planning (centralized) solution. Most papers focusing on the effects of random shocks on macroeconomic dynamics analyze the centralized solution where all externalities are internalized by the social planner; we will instead focus on the decentralized solution since it is likely to provide us with a more realistic characterization of real world dynamics. In a competitive solution, since agents do not take into account the 
existence of the pollution externality, the optimal control problem is concave such that first order conditions turn out to be also sufficient. However, even if the household does not internalize the externality this will affect macroeconomic dynamics, allowing us to take track of the aggregate effects of pollution. Hence, assuming that parameters $A_{t}$ and $B_{t}$ are considered as constants by utility maximizing households when deriving the first order conditions, similar steps to those in the study by La Torre et al. (2018)—which are briefly recalled in the Appendix - allow us to determine the following optimal policy functions:

$$
\begin{gathered}
c_{t}=[1-\beta \mathbb{E}(\alpha)] y_{t}, \\
u_{t}=\frac{[1-\beta \mathbb{E}(\phi)] \gamma_{t}}{[1-\beta \mathbb{E}(\phi)] \gamma_{t}+\beta \mathbb{E}(\gamma) \phi_{t}},
\end{gathered}
$$

where $\mathbb{E}(\alpha)=\sum_{i=1}^{m} p_{i} \alpha_{i}, \quad \mathbb{E}(\gamma)=\sum_{i=1}^{m} p_{i} \gamma_{i}, \quad$ and $\mathbb{E}(\phi)$ $=\sum_{i=1}^{m} p_{i} \phi_{i}$. Substituting (3) and (4) back into the law of motion of physical and human capital and taking into account the pollution-induced productivity levels $A_{t}=k_{t}^{\nu_{t} \chi_{t}} h_{t}^{\nu_{t} \omega_{t}}$ and $B_{t}=k_{t}^{\mu_{t} t_{t}} h_{t}^{\mu_{t} \omega_{t}}$ yield the optimal dynamics in the competitive economy, which turn out to be characterized by the following equations:

$$
\left\{\begin{array}{l}
k_{t+1}=\Delta_{t} z_{t} k_{t}^{\alpha_{t}+\nu_{t} \chi_{t}} h_{t}^{\gamma_{t}+\nu_{t} \omega_{t}} \\
h_{t+1}=\Theta_{t} \eta_{t} k_{t}^{\mu_{t} \gamma_{t}} h_{t}^{\phi_{t}+\mu_{t} \omega_{t}}
\end{array}\right.
$$

with

$$
\begin{gathered}
\Delta_{t}=\beta \mathbb{E}(\alpha)\left\{\frac{[1-\beta \mathbb{E}(\phi)] \gamma_{t}}{[1-\beta \mathbb{E}(\phi)] \gamma_{t}+\beta \mathbb{E}(\gamma) \phi_{t}}\right\}^{\gamma_{t}}, \\
\Theta_{t}=\left\{\frac{\beta \mathbb{E}(\gamma) \phi_{t}}{[1-\beta \mathbb{E}(\phi)] \gamma_{t}+\beta \mathbb{E}(\gamma) \phi_{t}}\right\}^{\phi_{t}} .
\end{gathered}
$$

\section{LOG-TRANSFORMATION}

We can apply the same technique developed in the study by La Torre et al. (2018) to build a specific transformation that recasts system (5) into an affine, topologically equivalent system. Specifically, we wish to transform it into a system of the following form:

$$
\left\{\begin{array}{l}
\varphi_{t+1}=\left(\alpha_{t}+\nu_{t} \chi_{t}\right) \varphi_{t}+\left(\gamma_{t}+\nu_{t} \omega_{t}\right) \psi_{t}+\zeta_{t} \\
\psi_{t+1}=\mu_{t} \chi_{t} \varphi_{t}+\left(\phi_{t}+\mu_{t} \omega_{t}\right) \psi_{t}+\vartheta_{t}
\end{array}\right.
$$

where the coefficients are the exponents of physical and human capital in our original Eq. (5) plus the parameters characterizing pollution externalities, and the additive random vector $\left(\zeta_{t}, \vartheta_{t}\right) \in \mathbb{R}^{2}$ takes on $m$ values corresponding to realizations of the multiplicative shocks $\left(z_{t}, \eta_{t}\right)$. We will show that, by imposing some conditions on the parameters, there exists a one-to-one continuous transformation from the dynamics of $\left(k_{t}, h_{t}\right)$ defined by (5) to those of $\left(\varphi_{t}, \psi_{t}\right)$ as in (8). By recalling Sec. II, the convergence of the random dynamical system in (8) to the steady state can be obtained by noticing that Eq. (8) is the chaos game associated with an
IFS with probabilities whose associated Markov operator will be converging to an invariant measure $\bar{\mu}$.

It may be useful to rewrite (8) in vector terms as follows:

$$
\left[\begin{array}{c}
\varphi_{t+1} \\
\psi_{t+1}
\end{array}\right]=\left[\begin{array}{cc}
\alpha_{t}+\nu_{t} \chi_{t} & \gamma_{t}+\nu_{t} \omega_{t} \\
\mu_{t} \chi_{t} & \phi_{t}+\mu_{t} \omega_{t}
\end{array}\right]\left[\begin{array}{l}
\varphi_{t} \\
\psi_{t}
\end{array}\right]+\left[\begin{array}{c}
\zeta_{t} \\
\vartheta_{t}
\end{array}\right],
$$

where

$$
Q_{t}=\left[\begin{array}{cc}
\alpha_{t}+\nu_{t} \chi_{t} & \gamma_{t}+\nu_{t} \omega_{t} \\
\mu_{t} \chi_{t} & \phi_{t}+\mu_{t} \omega_{t}
\end{array}\right]
$$

is a random $2 \times 2$ matrix which, together with the vector $\left(\zeta_{t}, \vartheta_{t}\right) \in \mathbb{R}^{2}$, takes on $m$ values corresponding to the $m$ shock realizations.

Proposition 1. There exists a one-to-one logarithmic transformation $\left(k_{t}, h_{t}\right) \rightarrow\left(\varphi_{t}, \psi_{t}\right)$ defined by

$$
\left\{\begin{array}{l}
\varphi_{t}=\rho_{1} \ln k_{t}+\rho_{2} \ln h_{t}+\rho_{3} \\
\psi_{t}=\rho_{4} \ln k_{t}+\rho_{5} \ln h_{t}+\rho_{6}
\end{array}\right.
$$

that is topologically conjugate to the nonlinear system (5) provided that the model's parameters $z_{i}, \eta_{i}, \alpha_{i}, \gamma_{i}, \phi_{i}, \nu_{i}, \mu_{i}, \chi_{i}, \omega_{i}$, the constants $\zeta_{i}, \vartheta_{i}$ in the IFS (8), together with the coefficients $\rho_{1}, \rho_{2}, \rho_{3}, \rho_{4}, \rho_{5}, \rho_{6}$, satisfy the following conditions:

$$
\left\{\begin{array}{l}
\left(\mu_{i} \chi_{i}\right) \rho_{2}=\left(\gamma_{i}+\nu_{i} \omega_{i}\right) \rho_{4} \\
\left(\gamma_{i}+\nu_{i} \omega_{i}\right) \rho_{1}=\left(\alpha_{i}+\nu_{i} \chi_{i}-\phi_{i}-\mu_{i} \omega_{i}\right) \rho_{2}+\left(\gamma_{i}+\nu_{i} \omega_{i}\right) \rho_{5} \\
\left(\alpha_{i}+\nu_{i} \chi_{i}-\phi_{i}-\mu_{i} \omega_{i}\right) \rho_{4}+\mu_{i} \chi_{i} \rho_{5}=\mu_{i} \chi_{i} \rho_{1} \\
\left(\ln \Delta_{i}+\ln z_{i}\right) \rho_{1}+\left(\ln \Theta_{i}+\ln \eta_{i}\right) \rho_{2}+\left(1-\alpha_{i}-\nu_{i} \chi_{i}\right) \rho_{3} \\
\quad-\left(\gamma_{i}+\nu_{i} \omega_{i}\right) \rho_{6}=\zeta_{i} \\
\left(\ln \Delta_{i}+\ln z_{i}\right) \rho_{4}+\left(\ln \Theta_{i}+\ln \eta_{i}\right) \rho_{5}+\left(1-\phi_{i}-\mu_{i} \omega_{i}\right) \rho_{6} \\
\quad-\mu_{i} \chi_{i} \rho_{3}=\vartheta_{i}
\end{array}\right.
$$

for all $i=1, \ldots, m$.

Proof. We use (11) to rewrite both sides of (8)

$$
\begin{aligned}
\rho_{1} \ln k_{t+1}+\rho_{2} \ln h_{t+1}+\rho_{3}= & \left(\alpha_{t}+\nu_{t} \chi_{t}\right)\left(\rho_{1} \ln k_{t}+\rho_{2} \ln h_{t}+\rho_{3}\right) \\
& +\left(\gamma_{t}+\nu_{t} \omega_{t}\right)\left(\rho_{4} \ln k_{t}+\rho_{5} \ln h_{t}\right. \\
& \left.+\rho_{6}\right)+\zeta_{t}, \rho_{4} \ln k_{t+1}+\rho_{5} \ln h_{t+1}+\rho_{6} \\
= & \mu_{t} \chi_{t}\left(\rho_{1} \ln k_{t}+\rho_{2} \ln h_{t}+\rho_{3}\right) \\
& +\left(\phi_{t}+\mu_{t} \omega_{t}\right)\left(\rho_{4} \ln k_{t}+\rho_{5} \ln h_{t}\right. \\
& \left.+\rho_{6}\right)+\vartheta_{t} .
\end{aligned}
$$

Then, use (5) to rewrite the LHS in each equation above in order to obtain the following two equations:

$$
\begin{aligned}
\rho_{1} \ln \Delta_{t} & +\rho_{1} \ln z_{t}+\rho_{1}\left(\alpha_{t}+\nu_{t} \chi_{t}\right) \ln k_{t}+\rho_{1}\left(\gamma_{t}+\nu_{t} \omega_{t}\right) \ln h_{t} \\
+ & \rho_{2} \ln \Theta_{t}+\rho_{2} \ln \eta_{t}+\rho_{2} \mu_{t} \chi_{t} \ln k_{t}+\rho_{2}\left(\phi_{t}+\mu_{t} \omega_{t}\right) \ln h_{t}+\rho_{3} \\
= & \left(\alpha_{t}+\nu_{t} \chi_{t}\right) \rho_{1} \ln k_{t}+\left(\alpha_{t}+\nu_{t} \chi_{t}\right) \rho_{2} \ln h_{t}+\left(\alpha_{t}+\nu_{t} \chi_{t}\right) \rho_{3} \\
& +\left(\gamma_{t}+\nu_{t} \omega_{t}\right) \rho_{4} \ln k_{t}+\left(\gamma_{t}+\nu_{t} \omega_{t}\right) \rho_{5} \ln h_{t} \\
& +\left(\gamma_{t}+\nu_{t} \omega_{t}\right) \rho_{6}+\zeta_{t}
\end{aligned}
$$




$$
\begin{aligned}
& \rho_{4} \ln \Delta_{t}+\rho_{4} \ln z_{t}+\rho_{4}\left(\alpha_{t}+\nu_{t} \chi_{t}\right) \ln k_{t}+\rho_{4}\left(\gamma_{t}+\nu_{t} \omega_{t}\right) \ln h_{t} \\
& +\rho_{5} \ln \Theta_{t}+\rho_{5} \ln \eta_{t}+\rho_{5} \mu_{t} \chi_{t} \ln k_{t}+\rho_{5}\left(\phi_{t}+\mu_{t} \omega_{t}\right) \ln h_{t}+\rho_{6} \\
& =\mu_{t} \chi_{t} \rho_{1} \ln k_{t}+\mu_{t} \chi_{t} \rho_{2} \ln h_{t}+\mu_{t} \chi_{t} \rho_{3} \\
& \quad+\left(\phi_{t}+\mu_{t} \omega_{t}\right) \rho_{4} \ln k_{t}+\left(\phi_{t}+\mu_{t} \omega_{t}\right) \rho_{5} \ln h_{t} \\
& \quad+\left(\phi_{t}+\mu_{t} \omega_{t}\right) \rho_{6}+\vartheta_{t} .
\end{aligned}
$$

As these equations must hold for all $t \geq 0$, under the i.i.d. assumption it is sufficient that they hold for all parameters' values, that is, for all $i=1, \ldots, m$; hence, in the sequel we replace the time index $t$ of each term involving only the model's parameters with the index $i=1, \ldots, m$ (clearly, the state variables $k_{t}$ and $h_{t}$ remain indexed by $t$ ). By equating the corresponding coefficients in the LHS and the RHS, Eqs. (13) and (14) become independent of values taken by the variables $\ln k_{t}$ and $\ln h_{t}$; this is equivalent to the following conditions (corresponding to system (25) in the study by La Torre et al., 2018):

$$
\left\{\begin{array}{l}
\left(\mu_{i} \chi_{i}\right) \rho_{2}=\left(\gamma_{i}+\nu_{i} \omega_{i}\right) \rho_{4} \\
\left(\gamma_{i}+\nu_{i} \omega_{i}\right) \rho_{1}=\left(\alpha_{i}+\nu_{i} \chi_{i}-\phi_{i}-\mu_{i} \omega_{i}\right) \rho_{2}+\left(\gamma_{i}+\nu_{i} \omega_{i}\right) \rho_{5} \\
\left(\alpha_{i}+\nu_{i} \chi_{i}-\phi_{i}-\mu_{i} \omega_{i}\right) \rho_{4}+\mu_{i} \chi_{i} \rho_{5}=\mu_{i} \chi_{i} \rho_{1}
\end{array}\right.
$$

for all $i=1, \ldots, m$.

After joining them with the terms that do not depend on $\ln k_{t}$ and $\ln h_{t}$ left in Eqs. (13) and (14) we obtain the system of $5 \mathrm{~m}$ equations in (12) and the proof is complete.
System (12) has $5 m$ equations and 6 unknowns, $\rho_{1}, \rho_{2}, \rho_{3}, \rho_{4}, \rho_{5}, \rho_{6}$; clearly such a system has no solution whenever there is more than one state of nature, i.e., when $m \geq 2$, as already with $m=2$ it has 10 equations in 6 unknowns. Hence, following an approach similar to that pursued in the study by La Torre et al. $(2015 ; 2018)$, in Sec. V we will add more constraints on the parameters' values so to increase the number of unknowns; specifically we will treat some values for the coefficients $z_{i}, \eta_{i}$ as unknowns in order to have as many unknowns as the number of equations in (12). A complication ensuing in this case is that system (12) ceases to be linear, as the new unknowns (even in their logexpression) $\ln z_{i}, \ln \eta_{i}$ enter multiplicatively the other unknowns of the type $\rho_{i}$. Therefore, to solve (12) we will rely on numerical methods.

\section{SOME SPECIFIC PARAMETERIZATIONS}

We now present two different model's parameterizations. The first allow us to generate exactly the classical Barnsley's fern, but this requires to impose somehow questionable parameter values. The second tries to fix this issue by focusing on more realistic parameter values; this however gives rise to an attractor very different from a fern.

\section{A. A model generating the classical Barnsley's fern}

It is well known that the classical Barnsley's fern (Barnsley, 1993) is produced by the following affine IFS:

$$
\begin{cases}w_{1}(\varphi, \psi)=\left[\begin{array}{cc}
0 & 0 \\
0 & 0.16
\end{array}\right]\left[\begin{array}{l}
\varphi \\
\psi
\end{array}\right]+\left[\begin{array}{l}
0 \\
0
\end{array}\right] \quad \text { with prob. } p_{1}=0.01 \\
w_{2}(\varphi, \psi)=\left[\begin{array}{ll}
-0.15 & 0.28 \\
0.26 & 0.24
\end{array}\right]\left[\begin{array}{l}
\varphi \\
\psi
\end{array}\right]+\left[\begin{array}{c}
0 \\
0.44
\end{array}\right] & \text { with prob. } p_{2}=0.07 \\
w_{3}(\varphi, \psi)=\left[\begin{array}{cc}
0.20 & -0.26 \\
0.23 & 0.22
\end{array}\right]\left[\begin{array}{l}
\varphi \\
\psi
\end{array}\right]+\left[\begin{array}{c}
0 \\
1.60
\end{array}\right] & \text { with prob. } p_{3}=0.07 \\
w_{4}(\varphi, \psi)=\left[\begin{array}{cc}
0.85 & 0.04 \\
-0.04 & 0.85
\end{array}\right]\left[\begin{array}{l}
\varphi \\
\psi
\end{array}\right]+\left[\begin{array}{c}
0 \\
1.60
\end{array}\right] & \text { with prob. } p_{4}=0.85\end{cases}
$$

We now look for a configuration of parameters' values for the economic growth model discussed in Sec. III such that the dynamics described by the IFS (15), generating the Barnsley's fern through the chaos game, are obtained as the result of some logarithmic transformation of the form in (11) applied to the optimal nonlinear dynamics defined by (5). In other words, following the arguments developed in Sec. IV we look for suitable values of the parameters $z_{i}, \eta_{i}, \alpha_{i}, \gamma_{i}, \phi_{i}, \nu_{i}, \mu_{i}, \chi_{i}, \omega_{i}$ that allow the existence of coefficients $\rho_{1}, \rho_{2}, \rho_{3}, \rho_{4}, \rho_{5}, \rho_{6}$ solving system (12). Note that the existence of a pollution externality allows some of the parameters in the random matrix (10) to take on negative values, which is specifically required in order to generate the IFS (15).

The realization of the random matrix $Q_{1}$ representing the first map in the IFS (15), $w_{1}$, imposes a constraint that yields immediately the values of $\rho_{2}, \rho_{4}$ and $\rho_{5}$ for all $i=1, \ldots, 4$. In fact, according to $(10), \mu_{1} \chi_{1}=0$ must hold for $i=1$; as $\left(\gamma_{i}+\nu_{i} \omega_{i}\right) \neq 0$ in the $w_{i}$ maps for $i=2, \ldots, 4$, replacing $\mu_{1} \chi_{1}=0$ in the first equation of system (12) for $i=1$ yields $\rho_{4}=0$, which, when substituted in the third equation of system (12) leads to $\mu_{i} \chi_{i} \rho_{5}=\mu_{i} \chi_{i} \rho_{1}$, which, in 
turn implies that $\rho_{5}=\rho_{1}$ must hold, as $\mu_{i} \chi_{i} \neq 0$ in the $w_{i}$ maps for $i=2, \ldots, 4$. Finally, using $\rho_{5}=\rho_{1}$ in the second set of equations of system (12) and noting that, besides having $\left(\gamma_{i}+\nu_{i} \omega_{i}\right) \neq 0$ in the $w_{i}$ maps for $i=2, \ldots, 4$, $\left(\alpha_{i}+\nu_{i} \chi_{i}-\phi_{i}-\mu_{i} \omega_{i}\right) \neq 0$ at least in the $w_{1}$ map (for $i=1$ ), we observe that also $\rho_{2}=0$ must hold. Therefore, with $\rho_{2}=\rho_{4}=0$ and $\rho_{5}=\rho_{1}$ satisfying the first three equations in (12) for all $i=1, \ldots, 4$, after substituting such values in the remaining equations the whole system boils down to the following 8 equations:

$$
\left\{\begin{array}{l}
\left(\ln \Delta_{i}+\ln z_{i}\right) \rho_{1}+\left(1-\alpha_{i}-\nu_{i} \chi_{i}\right) \rho_{3}-\left(\gamma_{i}+\nu_{i} \omega_{i}\right) \rho_{6}=\zeta_{i} \\
\left(\ln \Theta_{i}+\ln \eta_{i}\right) \rho_{1}+\left(1-\phi_{i}-\mu_{i} \omega_{i}\right) \rho_{6}-\mu_{i} \chi_{i} \rho_{3}=\vartheta_{i}
\end{array} \quad \text { for } i=1, \ldots, 4\right.
$$

having only the three coefficients $\rho_{1}, \rho_{3}$, and $\rho_{6}$ as unknowns.

As anticipated at the end of Sec. IV, we will choose arbitrary 3 out of the 8 values for the multiplicative shocks $z_{i}, \eta_{i}$ (or, equivalently, $\ln z_{i}, \ln \eta_{i}$ ) and leave the remaining 5 values for $z_{i}, \eta_{i}\left(\ln z_{i}, \ln \eta_{i}\right)$ as unknowns so that, together with the 3 coefficients $\rho_{1}, \rho_{3}, \rho_{6}$, (16) becomes a system of 8 equations in 8 unknowns. Specifically, we will set

$$
\ln z_{1}=-0.4, \quad \ln \eta_{1}=-0.2, \quad \text { and } \quad \ln \eta_{2}=0
$$

and leave $\ln z_{2}, \ln z_{3}, \ln z_{4}, \ln \eta_{3}$, and $\ln \eta_{4}$ as unknowns, to be found as part of the solution for system (16).

As far as all other parameters are concerned, we set $\beta=0.96$, while the constant terms $\zeta_{i}$ and $\vartheta_{i}$ in system (16) are clearly given by the coordinates of the constant vectors in the maps $w_{i}$ of the IFS (15). To choose all the exponents involved in the optimal dynamics (5), we must equate the random matrix $Q_{i}$ defined in (10) to the 4 values considered in the IFS (15), that is, we must choose values for the exponents $\alpha_{i}, \gamma_{i}, \phi_{i}, \nu_{i}, \mu_{i}, \chi_{i}, \omega_{i}$ that satisfy the following 4 sets of conditions:

$$
\begin{aligned}
& \text { 1. }\left\{\begin{array}{l}
\alpha_{1}+\nu_{1} \chi_{1}=0 \\
\gamma_{1}+\nu_{1} \omega_{1}=0 \\
\mu_{1} \chi_{1}=0 \\
\phi_{1}+\mu_{1} \omega_{1}=0.16
\end{array}\right. \\
& \text { 3. }\left\{\begin{array} { l } 
{ \alpha _ { 3 } + \nu _ { 3 } \chi _ { 3 } = 0 . 2 0 } \\
{ \gamma _ { 3 } + \nu _ { 3 } \omega _ { 3 } = - 0 . 2 6 } \\
{ \mu _ { 3 } \chi _ { 3 } = 0 . 2 3 } \\
{ \phi _ { 3 } + \mu _ { 3 } \omega _ { 3 } = 0 . 2 2 }
\end{array} \quad \text { 4. } \left\{\begin{array}{l}
\alpha_{2}+\nu_{2} \chi_{2}=-0.15 \\
\gamma_{2}+\nu_{2} \omega_{2}=0.28 \\
\mu_{2} \chi_{2}=0.26 \\
\phi_{2}+\mu_{2} \omega_{2}=0.24
\end{array}\right.\right. \\
&
\end{aligned}
$$

1. The third equation for the first shock realization requires that either $\mu_{1}$ or $\chi_{1}$ be zero; we opt for the former solution, $\mu_{1}=0$, as the latter would imply that $\alpha_{1}$ is zero as well from the first equation, which is ruled out by the basic assumptions on the model's fundamentals. Such choice implies that, in the first shock realization, the production of human capital is not being affected by pollution. Setting $\alpha_{1}=0.3$ and $\nu_{1}=-0.5$ (output production decreases in the stock of pollution), the first equation yields $\chi_{1}=-0.3 /(-0.5)=0.6$ (pollution increases in the stock of physical capital); setting $\gamma_{1}=0.6$, the second equation yields $\omega_{1}=-0.6 /(-0.5)=1.2 \quad$ (pollution increases in the stock of human capital as well); finally, with $\mu_{1}=0$ the last equation implies that $\phi_{1}=0.16$. This configuration envisages $\chi_{1}<\omega_{1}$, so that human capital turns out to be more pollution-intense than physical capital.

2. In the second set of conditions, we set $\mu_{2}=-0.5$ (human capital production decreases in the stock of pollution), so that the third equation yields $\chi_{2}=0.26 /(-0.5)=-0.52$ (pollution decreases in the stock of physical capital), which, by setting $\alpha_{2}=0.15$ in the first equation, leads to $\nu_{2}=(-0.15-0.15) /(-0.52)=0.3 /(0.52)=0.577$ (output production increases in the stock of pollution). By setting $\phi_{2}=0.1$, the last equation yields $\omega_{2}=(0.24$ $-0.1) /(-0.5)=0.14 /(-0.5)=-0.28$ (pollution decreases in the stock of human capital), which, after substituting into the second equation, in turn yields $\gamma_{2}=0.28$ $-0.577(-0.28)=0.28+0.162=0.442$. In this case again $\chi_{2}<\omega_{2}$, but now both have negative values, which implies that the stock of pollution is more sensitive to changes in the stock of physical capital than in the stock of human capital.

3. In the third set of conditions again we set $\mu_{3}=-0.5$ (human capital production decreases in the stock of pollution), so that the third equation yields $\chi_{3}=0.23 /(-0.5)$ $=-0.46$ (pollution decreases in the stock of physical capital), which, by setting $\alpha_{3}=0.05$ in the first equation, leads to $\nu_{3}=(0.20-0.05) /(-0.46)=0.15 /(-0.46)=-0.326$ (output production decreases in the stock of pollution). By setting $\gamma_{3}=0.05$, the second equation yields $\omega_{3}$ $=(-0.26-0.05) /(-0.326)=0.31 /(0.326)=0.951 \quad($ pollution increases in the stock of human capital), which, after substituting into the last equation, in turn yields $\phi_{3}=0.22-(-0.5)(0.951)=0.22+0.475=0.695$. Also in this scenario $\chi_{3}<\omega_{3}$, but now $\chi_{3}$ and $\omega_{3}$ have opposite signs, meaning that they affect pollution in opposite directions.

4. In the fourth shock realization, we set $\mu_{4}=-0.1$ (human capital production decreases in the stock of pollution), so that the third equation yields $\chi_{4}=-0.04 /(-0.1)=0.4$ (pollution increases in the stock of physical capital), which, by setting $\alpha_{4}=0.89$ in the first equation, leads to $\nu_{4}=(0.85-0.89) /(0.4)=-0.04 /(0.4)=-0.1$ (output production decreases in the stock of pollution with the 
TABLE I. Parameters characterizing our model, additive constants, and probability values corresponding to the IFS (15) generating the classical Barnsley's (1993) fern.

\begin{tabular}{llllccccccc}
\hline \hline$i$ & $\alpha_{i}$ & $\gamma_{i}$ & $\phi_{i}$ & $\nu_{\mathrm{i}}$ & $\mu_{\mathrm{i}}$ & $\chi_{\mathrm{i}}$ & $\omega_{\mathrm{i}}$ & $\zeta_{\mathrm{i}}$ & $\vartheta_{i}$ & $p_{i}$ \\
\hline 1 & 0.3 & 0.6 & 0.16 & -0.5 & 0 & 0.6 & 1.2 & 0 & 0 & 0.01 \\
2 & 0.15 & 0.442 & 0.1 & 0.557 & -0.5 & -0.52 & -0.28 & 0 & 0.44 & 0.07 \\
3 & 0.05 & 0.05 & 0.695 & -0.326 & -0.5 & -0.46 & 0.951 & 0 & 1.60 & 0.07 \\
4 & 0.89 & 0.06 & 0.87 & -0.1 & -0.1 & 0.4 & 0.2 & 0 & 1.60 & 0.85 \\
\hline \hline
\end{tabular}

same intensity as human capital production). By setting $\gamma_{4}$ $=0.06$, the second equation yields $\omega_{4}=(0.04-0.06) /$ $(-0.1)=-0.02 /(-0.1)=0.2$ (pollution increases in the stock of human capital), which, after substituting into the last equation, in turn yields $\phi_{4}=0.85-(-0.1)(0.2)$ $=0.85+0.02=0.87$. The fourth configuration thus has $\chi_{4}>\omega_{4}$, so that physical capital turns out to be more pollution-intense than human capital.

Table I summarizes all parameters' values discussed so far.

Under these parameters' choices

$$
\begin{aligned}
& \mathbb{E}(\alpha)=\sum_{i=1}^{4} \alpha_{i} p_{i}=0.7735, \quad \mathbb{E}(\gamma)=\sum_{i=1}^{4} \gamma_{i} p_{i}=0.0914 \\
& \text { and } \mathbb{E}(\phi)=\sum_{i=1}^{4} \phi_{i} p_{i}=0.7968,
\end{aligned}
$$

such that the coefficients (6) and (7) defining the optimal policy of the model at the end of Sec. III become

\begin{tabular}{|c|cc|}
\hline$i$ & $\Delta_{i}$ & $\Theta_{i}$ \\
\hline 1 & 0.7015 & 0.6809 \\
2 & 0.7164 & 0.7747 \\
3 & 0.6779 & 0.8847 \\
4 & 0.6642 & 0.8629 \\
\hline
\end{tabular}

Recall that, following the discussion at the beginning of this section on the parameters' constraints due to the first shock realization, $\rho_{2}=\rho_{4}=0$ and $\rho_{1}=\rho_{5}$. Hence, using the choice in (17) for the values of $\ln z_{1}, \ln \eta_{1}, \ln \eta_{2}$, we find the following unique solution for system (16) by means of the standard (symbolic, not numerical) 'solve' routine in Maple

$$
\begin{aligned}
& \rho_{1}=\rho_{5}=5.6984 \\
& \rho_{2}=\rho_{4}=0 \\
& \rho_{3}=4.3 \\
& \rho_{6}=3.9638,
\end{aligned}
$$

with all multiplicative shock configurations

\begin{tabular}{|c|cc|}
\hline$i$ & $z_{i}$ & $\eta_{i}$ \\
\hline 1 & 0.6703 & 0.8187 \\
2 & 0.7123 & 1 \\
3 & 0.6731 & 1.0345 \\
4 & 1.3824 & 1.3414 \\
\hline
\end{tabular}

where the values of $z_{1}, \eta_{1}$, and $\eta_{2}$ correspond to the choice in (17) and all other values are found as a solution of (16).

Figure 1(a) reports the standard Barnsley's fern by tracing 50,000 random iterations (the Maple code is available from the authors upon request) of the affine IFS defined in (15) expressed in terms of the log-transformed variables $\varphi, \psi$. It is well known that the unique invariant measure supported on the fern is singular (Theorem 1 in La Torre et al., 2018). Figure 1(b) portraits the attractor of the corresponding nonlinear IFS defined in (5) for the parameters' values characterizing our growth model reported in Table I, expressed in terms of physical and human capital, $k, h$; such attractor turns out to be a downsized version of the fern in Fig. 1(a) (rescaled by a factor 10 on the horizontal axis and by a factor 4 on the vertical axis) which is being translated into the positive orthant ( $k$ and $h$ cannot be negative) and somewhat straighten up along its main branch.

Some comments on the parametrization employed to generate the classical fern are needed. Leaving aside the

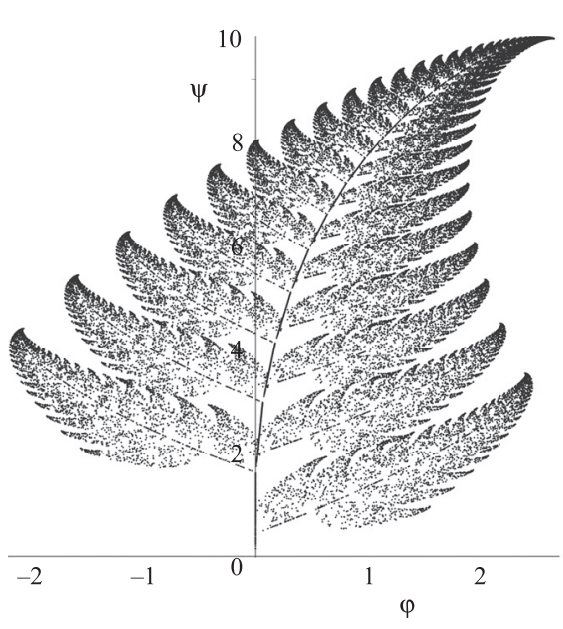

(a)

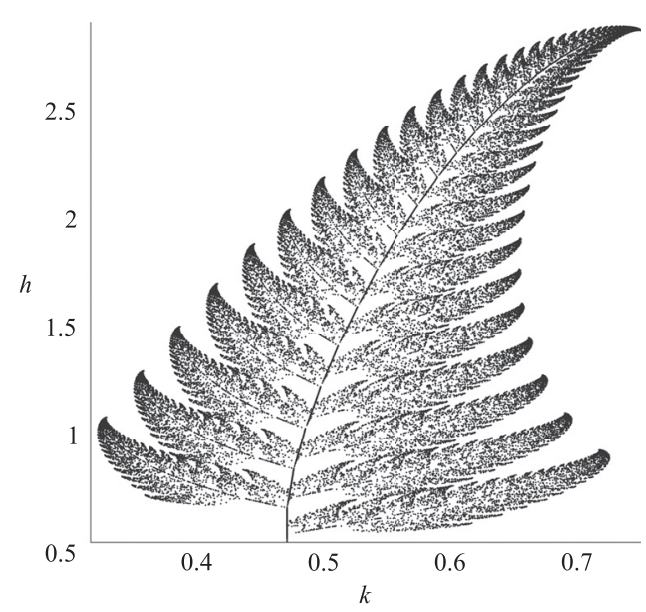

(b)
FIG. 1. Approximation through 50,000 random iterations of (a) the IFS in (15) generating the standard Barnsley's fern as attractor and (b) the attractor of the corresponding nonlinear IFS in (5) for the parameters' values listed in Table I. 
productivity parameter values in (18), the four-shocks parameters' configuration reported in Table I does not only clearly satisfy all the assumptions on the model's fundamentals, but may even be considered not totally unrealistic, as the following tentative arguments try to clarify.

The first scenario foresees reasonable values for all three factor shares (perhaps the human capital share, $\phi_{1}$, in human capital production is too low), an output production function which decreases in the stock of pollution $\left(\nu_{1}<0\right)$, while the latter increases in both the physical and human capital stocks $\left(\chi_{1}, \omega_{1}>0\right)$. The only anomalies may appear to be an output production process that is not being affected by pollution $\left(\mu_{1}=0\right)$ and a human capital production that generates pollution with and intensity which is the double of that of physical capital $\left(\omega_{1}=2 \chi_{1}\right)$-i.e., human capital is a dirtier input than physical capital. The latter feature may be characteristic in an economy that is more efficient in producing physical goods than in recycling waste generated by human activity.

In the second shock configuration, besides perhaps too low values for the physical and human capital shares in output production and a human capital share in human capital production even lower than in the first case, the most apparent anomalies are given by a positive relationship between the pollution stock and output production $\left(\nu_{2}>0\right)$-i.e., pollution becomes itself some exogenous production factortogether with a negative relationship between both physical and human capital and pollution stock $\left(\chi_{2}, \omega_{2}<0\right)$-i.e., physical and human capital accumulation reduces pollution. On the other hand, the negative relationship between pollution and human capital $\left(\mu_{2}<0\right)$ has an intuitive and appealing interpretation if one attributes to the latter a broader meaning that includes health. Such a scenario may describe a virtuous economy that is capable of recycling pollution as a production enhancer $\left(\nu_{2}>0\right)$, while physical together with human capital accumulation may be thought as general progress capable of bringing about a better and more efficient treatment and recycling of polluting by-products.

The third shock environment is characterized by even smaller values for the physical and human capital shares in output production, while the human capital share in human capital production more realistically becomes larger. The pollution parameters envisage a reasonable negative relationship between the pollution stock and both physical and human capital production $\left(\nu_{3}, \mu_{3}<0\right)$ together with a positive relationship between human capital accumulation and pollution $\left(\omega_{3}>0\right)$, while, again, the relationship between physical capital accumulation and the pollution stock is negative $\left(\chi_{3}<0\right)$-i.e., physical capital accumulation reduces pollution. In this case, human capital turns out to be a dirty input, while physical capital accumulation has a beneficial effect on the environment. This may occur in an economy that invests a lot in renewable technologies.

The last exogenous shock includes an output production function with a very large physical capital share compared to the human capital share as well as a quite large human capital share in human capital production, while the pollution stock decreases both physical and human capital production $\left(\nu_{4}, \mu_{4}<0\right)$ and both physical and human capital accumulation increase the pollution stock $\left(\chi_{4}, \omega_{4}>0\right)$. Besides the excessive displacement in the final production factor shares (there is, however, a growing discussion on the reduction of the labor income share due to automation, machines and AI replacing labor in tasks that it used to perform, implying that scenarios envisaging very low intensities of human capital in final production may be well deemed plausible. See, e.g., Korinek and Stiglitz (2017) and Acemoglu and Restrepo (2018), who also propose policies aimed at countervailing such tendency and the inequality it involves), this scenario seems to describe a somewhat more realistic economy, as human capital happens to be a greener input than physical capital $\left(\omega_{4}<\chi_{4}\right)$.

\section{B. A tentative more realistic example}

Considering the possible drawbacks of our previous parametrization, we now rely on a set of parameter values allowing for a more intuitive interpretation of typical real world situations. Such a configuration is summarized in Table II.

To such a configuration corresponds the following affine IFS:

$$
\left\{\begin{array}{lll}
w_{1}(\varphi, \psi)=\left[\begin{array}{cc}
0.03 & 0.87 \\
-0.14 & -0.44
\end{array}\right]\left[\begin{array}{l}
\varphi \\
\psi
\end{array}\right]+\left[\begin{array}{l}
0 \\
0
\end{array}\right] & & \text { with prob. } p_{1}=0.15 \\
w_{2}(\varphi, \psi)=\left[\begin{array}{cc}
0.06 & 0.69 \\
-0.15 & -0.10
\end{array}\right]\left[\begin{array}{l}
\varphi \\
\psi
\end{array}\right]+\left[\begin{array}{c}
0 \\
0.44
\end{array}\right] & & \text { with prob. } p_{2}=0.30 \\
w_{3}(\varphi, \psi)=\left[\begin{array}{cc}
0.20 & 0.39 \\
-0.15 & 0.36
\end{array}\right]\left[\begin{array}{l}
\varphi \\
\psi
\end{array}\right]+\left[\begin{array}{c}
0 \\
1.60
\end{array}\right] & & \text { with prob. } p_{3}=0.50 \\
w_{4}(\varphi, \psi)=\left[\begin{array}{cc}
0.21 & 0.15 \\
0 & 0.70
\end{array}\right]\left[\begin{array}{l}
\varphi \\
\psi
\end{array}\right]+\left[\begin{array}{c}
0 \\
1.60
\end{array}\right] & & \text { with prob. } p_{4}=0.05
\end{array}\right.
$$

In order to solve the first $3 m$ equations in system (12) in one shot, we exploit the same property that we used at the beginning of Sec. V A of having zero as the left bottom element in one of the four stochastic matrices $Q_{i}$. Specifically, 
TABLE II. Factor shares, additive constants and probability values corresponding to the IFS (19).

\begin{tabular}{ccccccccccc}
\hline \hline$i$ & $\alpha_{i}$ & $\gamma_{i}$ & $\phi_{i}$ & $\nu_{i}$ & $\mu_{i}$ & $\chi_{i}$ & $\omega_{i}$ & $\zeta_{i}$ & $\vartheta_{i}$ & $p_{i}$ \\
\hline 1 & 0.05 & 0.94 & 0.05 & -0.1 & -0.7 & 0.2 & 0.7 & 0 & 0 & 0.15 \\
2 & 0.15 & 0.84 & 0.15 & -0.3 & -0.5 & 0.3 & 0.5 & 0 & 0.44 & 0.30 \\
3 & 0.45 & 0.54 & 0.45 & -0.5 & -0.3 & 0.5 & 0.3 & 0 & 1.60 & 0.50 \\
4 & 0.70 & 0.29 & 0.70 & -0.7 & 0 & 0.7 & 0.2 & 0 & 1.60 & 0.05 \\
\hline \hline
\end{tabular}

we set $\mu_{4}=0$ for $i=4$, so that in the fourth shock realization the production of human capital is not being affected by pollution. The same argument followed in Sec. V A then immediately yields $\rho_{2}=\rho_{4}=0$ and $\rho_{5}=\rho_{1}$ satisfying the first three equations in (12) for all $i=1, \ldots, 4$; after substituting such values in the remaining equations the whole system again boils down to the same 8 equations as in (16) having only the three coefficients $\rho_{1}, \rho_{3}$, and $\rho_{6}$ as unknowns. To have 8 unknowns in total as well, again we choose arbitrary 3 out of the 8 values for the multiplicative shocks $z_{i}, \eta_{i}$ (or, equivalently, $\ln z_{i}, \ln \eta_{i}$ ) and leave the remaining 5 values for $z_{i}, \eta_{i}\left(\ln z_{i}, \ln \eta_{i}\right)$ as unknowns; in this example, we will set

$$
\ln z_{1}=\ln \eta_{1}=\ln \eta_{2}=0
$$

and leave $\ln z_{2}, \ln z_{3}, \ln z_{4}, \ln \eta_{3}$, and $\ln \eta_{4}$ as unknowns, to be found as part of the solution for system (16).

We keep the individual discount factor value $\beta=0.96$ and all the values of the constant terms $\zeta_{i}$ and $\vartheta_{i}$ in system (12)_i.e., in the IFS (19)_-are the same as the coordinates of the constant vectors in the maps $w_{i}$ of the IFS (15). We choose four values for each factor share (the first three columns in Table II) that, together with the probabilities listed in the last column of Table II, on average resemble empirical evidence
Under these assumptions the coefficients (6) and (7) defining the optimal policy of the model at the end of Sec. III become

\begin{tabular}{|c|cc|}
\hline$i$ & $\Delta_{i}$ & $\Theta_{i}$ \\
\hline 1 & 0.2867 & 0.8583 \\
2 & 0.2637 & 0.7464 \\
3 & 0.2201 & 0.6886 \\
4 & 0.2133 & 0.7725 \\
\hline
\end{tabular}

As far as the four pollution parameters, $\nu_{i}, \mu_{i}, \chi_{i}, \omega_{i}$, are concerned, we propose an increasing pattern for both the (absolute value of the) intensity of pollution affecting output production, $\nu_{i}$, and the intensity of physical capital accumulation adding to the pollution stock, $\chi_{i}$, as the physical capital share $\alpha_{i}$ in output production increases; similarly, also the (absolute value of the) intensity of pollution affecting human capital production, $\mu_{i}$, and the intensity of human capital accumulation adding to the pollution stock, $\omega_{i}$, increase as the human capital share $\gamma_{i}$ in human capital production increases. The latter property is consistent with the assumption that in the fourth shock configuration pollution does not affect the production of human capital, $\mu_{4}=0$, for the technical reasons explained before.

Having set $\rho_{2}=\rho_{4}=0$ and $\rho_{1}=\rho_{5}$ and using the choice in (20) for the values of $\ln z_{1}, \ln \eta_{1}, \ln \eta_{2}$, we find the following unique solution for system (16) by means of the standard (symbolic, not numerical) "solve" routine in Maple

$$
\begin{aligned}
& \mathbb{E}(\alpha)=\sum_{i=1}^{4} \alpha_{i} p_{i}=\sum_{i=1}^{4} \phi_{i} p_{i}=0.3125, \quad \text { and } \\
& \mathbb{E}(\gamma)=\sum_{i=1}^{4} \gamma_{i} p_{i}=0.6775 .
\end{aligned}
$$

$$
\begin{aligned}
& \rho_{1}=\rho_{5}=-3.6368, \\
& \rho_{2}=\rho_{4}=0, \\
& \rho_{3}=-4.6267, \\
& \rho_{6}=0.0640,
\end{aligned}
$$

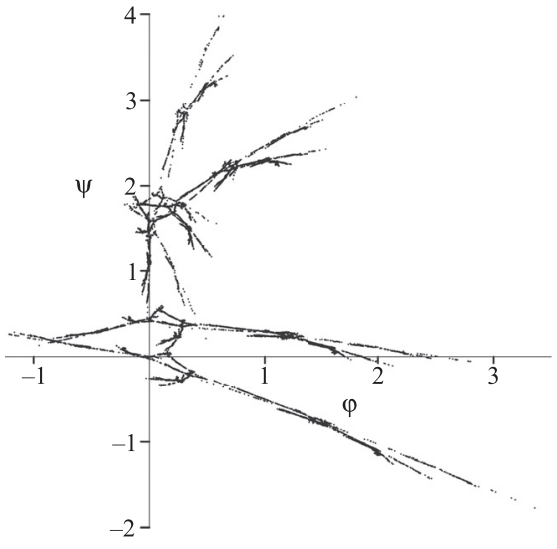

(a)

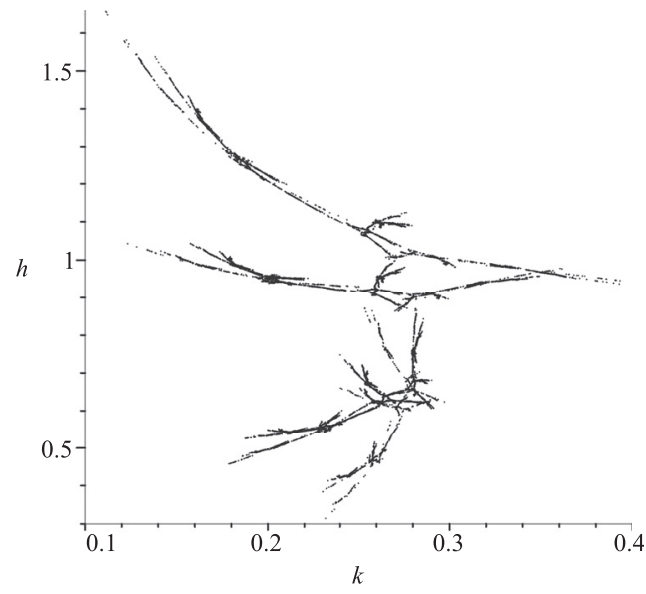

(b)
FIG. 2. Approximation through 50,000 random iterations of (a) the attractor of the affine IFS in (19) and (b) the attractor of the corresponding nonlinear IFS in (5) for the parameters' values listed in Table II. 
with all multiplicative shocks configurations

\begin{tabular}{|c|cc|}
\hline$i$ & $z_{i}$ & $\eta_{i}$ \\
\hline 1 & 1 & 1 \\
2 & 1.1331 & 1 \\
3 & 1.6307 & 0.7816 \\
4 & 1.7117 & 0.8381 \\
\hline
\end{tabular}

where the values of $z_{1}, \eta_{1}$, and $\eta_{2}$ correspond to the choice in (20) and all other values are found as a solution of (16).

Figure 2(a) traces 50,000 random iterations to approximate the attractor of the affine IFS (19) expressed in terms of the log-transformed variables $\varphi, \psi$. By applying condition (5) of Theorem 1 in the study by La Torre et al. (2018) to the random matrix $Q_{i}$ defined in (10), we find that also for the parameters' values reported in Table II the invariant measure supported on the attractor in Fig. 2(a) is singular, as

$$
\begin{gathered}
\left|\operatorname{det}\left(Q_{1}\right)\right|^{p_{1}}\left|\operatorname{det}\left(Q_{2}\right)\right|^{p_{2}}\left|\operatorname{det}\left(Q_{3}\right)\right|^{p_{3}}\left|\operatorname{det}\left(Q_{4}\right)\right|^{p_{4}} \\
\quad<p_{1}^{p_{1}} p_{2}^{p_{2}} p_{3}^{p_{3}} p_{4}^{p_{4}} \quad \Longleftrightarrow \quad 0.1170<0.3191 .
\end{gathered}
$$

Figure 2(b) portraits the attractor of the corresponding nonlinear IFS defined in (5) for the parameters' values characterizing our growth model reported in Table II, expressed in terms of physical and human capital, $k, h$; such an attractor turns out to be a downsized version of the fern in Fig. 2(a), which is being translated into the positive orthant ( $k$ and $h$ cannot be negative) and rotated by $180^{\circ}$, consistently with both the magnitude and the (negative) signs of the nonzero coefficients $\rho_{1}, \rho_{3}$, and $\rho_{5}$ in $(21)$.

\section{CONCLUSIONS}

We extend the analysis of the fractal nature of steady states in macroeconomic models, by considering a stochastic two-sector discrete-time economic growth model in which shocks affect the production function not only directly (via productivity and factor shares) but also indirectly (via a pollution externality). This extension is meaningful from both economic and mathematical points of view, since it allows capturing important economyenvironment feedback and it gives rise to an IFS with potential negative coefficients. This latter characteristic, completely lacking in the extant literature and due to the presence of the pollution externality, is essential in order to prove our main result: a full-fledged macroeconomic model can generate optimal dynamics which, through an appropriate log-transformation, converges to a singular measure supported on the classical Barnsley's fern. To the best of our knowledge, no other paper is able to generate the Barnsley's fern as an attractor of the optimal dynamics emerging from a meaningful macroeconomic model. It should be also emphasized that the key features of our model allowing for a representation of the logtransformed optimal dynamics by means of an IFS with potentially negative coefficients paves the way for possibly characterizing a quite large family of fractal attractors, including, perhaps, the renowned maple-leaf.

This paper closes an open gap in the stochastic growth and fractal attractor literature. Three important questions still remain open: (i) how to characterize also absolute continuity of the invariant measure in a twodimensional affine IFS; (ii) how to extend the analysis in a framework in which probability is not constant but place-dependent; and (iii) which other fractal attractors can be generated from the optimal dynamics of macroeconomic models. These further issues are on top of our future research agenda.

\section{APPENDIX: OPTIMAL POLICY CALCULATION IN SECTION III}

We first eliminate controls and keep only the two state variables by restating problem (1) in reduced-form

$$
\begin{aligned}
& \max _{\left\{k_{t}, h_{t}\right\}} \sum_{t=0}^{\infty} \beta^{t} \mathbb{E}_{0} \ln \left\{z_{t} A_{t} k_{t}^{\alpha_{t}}\left[h_{t}-\left(\frac{h_{t+1}}{\eta_{t} B_{t}}\right)^{\frac{1}{\phi_{t}}}\right]^{\gamma_{t}}-k_{t+1}\right\}, \\
& \text { s.t. }\left\{\begin{array}{l}
0 \leq k_{t+1} \leq z_{t} A_{t} k_{t}^{\alpha_{t}}\left[h_{t}-\left(\frac{h_{t+1}}{\eta_{t} B_{t}}\right)^{\frac{1}{\phi_{t}}}\right]^{\gamma_{t}} \\
0 \leq h_{t+1} \leq \eta_{t} B_{t} h_{t}^{\phi_{t}} \\
k_{0}>0, h_{0}>0,\left(z_{0}, \eta_{0}, \alpha_{0}, \gamma_{0}, \phi_{0}, A_{0}, B_{0}\right) \text { are given },
\end{array}\right.
\end{aligned}
$$

where, from the households point of view, $A_{t}=k_{t}^{\nu_{t} \chi_{t}} h_{t}^{\nu_{t} \omega_{t}}$ and $B_{t}=k_{t}^{\mu_{t} \chi_{t}} h_{t}^{\mu_{t} \omega_{t}}$ are taken as given. An argument similar to that used in Sec. III B of La Torre et al. (2018) applies here to establish that problem (A1) is concave.

The Euler-Lagrange equation with respect to $k_{t}$ is

$$
\begin{gathered}
z_{t-1} A_{t-1} k_{t-1}^{\alpha_{t-1}}\left[h_{t-1}-\left(\frac{h_{t}}{\eta_{t-1} B_{t-1}}\right)^{\frac{1}{\phi_{t-1}}}\right]^{\gamma_{t-1}}-k_{t} \\
+\beta \mathbb{E}_{t-1}\left\{\frac{z_{t} A_{t} \alpha_{t} k_{t}^{\alpha_{t}-1}\left[h_{t}-\left(\frac{h_{t+1}}{\eta_{t} B_{t}}\right)^{\frac{1}{\phi_{t}}}\right]^{\gamma_{t}}}{z_{t} A_{t} k_{t}^{\alpha_{t}}\left[h_{t}-\left(\frac{h_{t+1}}{\eta_{t} B_{t}}\right)^{\frac{1}{\phi_{t}}}\right]^{\gamma_{t}}-k_{t+1}}\right\}=0,
\end{gathered}
$$

which, by assuming that $k_{t+1}=s z_{t} A_{t} k_{t}^{\alpha_{t}}\left\{h_{t}-\left[h_{t+1} /\right.\right.$ $\left.\left.\left(\eta_{t} B_{t}\right)\right]^{1 / \phi_{t}}\right\}^{\gamma_{t}}$ for some constant $0<s<1$, and recalling that $\left\{\left(z_{t}, \eta_{t}, \alpha_{t}, \gamma_{t}, \phi_{t}, A_{t}, B_{t}\right)\right\}$ is an i.i.d. process, boils down to 


$$
\frac{1}{\frac{k_{t}}{s}-k_{t}}=\beta \mathbb{E}_{t-1}\left\{\frac{z_{t} A_{t} \alpha_{t} k_{t}^{\alpha_{t}-1}\left[h_{t}-\left(\frac{h_{t+1}}{\eta_{t} B_{t}}\right)^{\frac{1}{\phi_{t}}}\right]^{\gamma_{t}}}{z_{t} A_{t} k_{t}^{\alpha_{t}}\left[h_{t}-\left(\frac{h_{t+1}}{\eta_{t} B_{t}}\right)^{\frac{1}{\phi_{t}}}\right]^{\gamma_{t}}-s z_{t} A_{t} k_{t}^{\alpha_{t}}\left[h_{t}-\left(\frac{h_{t+1}}{\eta_{t} B_{t}}\right)^{\frac{1}{\phi_{t}}}\right]^{\gamma_{t}}}\right\}=\frac{\beta \mathbb{E}_{t-1}\left(\alpha_{t}\right)}{(1-s) k_{t}}=\frac{\beta \mathbb{E}_{(\alpha)}}{(1-s) k_{t}},
$$

where in the second equality $k_{t}$ has been pulled out of the expectation because, under our assumption, $k_{t}$ $=s z_{t-1} A_{t-1} k_{t-1}^{\alpha_{t-1}}\left\{h_{t-1}-\left[h_{t} /\left(\eta_{t-1} B_{t-1}\right)\right]^{1 / \phi_{t-1}}\right\}^{\gamma_{t-1}}$ is a deterministic choice taken at time $t-1$, with all the information available at that moment (including the optimal choice for $h_{t}$ ), and in the last equality we used the i.i.d. assumption on the random variable $\alpha_{t}$, so that $\mathbb{E}(\alpha)=\sum_{i=1}^{m} p_{i} \alpha_{i}$ is a constant. Then, the Euler-Lagrange equation becomes

$$
\frac{s}{(1-s) k_{t}}=\frac{\beta \mathbb{E}(\alpha)}{(1-s) k_{t}}
$$

so that the constant term is given by $s=\beta E(\alpha)$. Hence, given the optimal choice for the human capital $h_{t+1}$ (or, equivalently, $u_{t} h_{t}$ ), the (candidate) optimal policy for the physical capital is given by

$$
k_{t+1}=\beta \mathbb{E}(\alpha) z_{t} A_{t} k_{t}^{\alpha_{t}}\left[h_{t}-\left(\frac{h_{t+1}}{\eta_{t} B_{t}}\right)^{\frac{1}{\phi_{t}}}\right]^{\gamma_{t}}=\beta \mathbb{E}(\alpha) z_{t} A_{t} k_{t}^{\alpha_{t}}\left(u_{t} h_{t}\right)^{\gamma_{t}},
$$

where in the last equality we have recovered the original control formulation for human capital employed in final production. Then, from the constraint (2), the optimal consumption as in (3) is immediately obtained.

The Euler-Lagrange equation with respect to $h_{t}$ leads to

$$
\begin{gathered}
-\frac{z_{t-1} A_{t-1} \gamma_{t-1} k_{t-1}^{\alpha_{t-1}}\left[h_{t-1}-\left(\frac{h_{t}}{\eta_{t-1} B_{t-1}}\right)^{\frac{1}{\phi_{t-1}}}\right]^{\gamma_{t-1}-1} \frac{1}{\phi_{t-1}}\left(\frac{h_{t}}{\eta_{t-1} B_{t-1}}\right)^{\frac{1}{\phi_{t-1}}-1} \frac{1}{z_{t-1} A_{t-1} k_{t-1}^{\alpha_{t-1}}\left[h_{t-1}-\left(\frac{h_{t}}{\eta_{t-1} B_{t-1}}\right)^{\frac{1}{\phi_{t-1}}}\right]^{\gamma_{t-1}}-k_{t}}}{+\beta \mathbb{E}_{t-1}\left\{\frac{z_{t} A_{t} \gamma_{t} k_{t}^{\alpha_{t}}\left[h_{t}-\left(\frac{h_{t+1}}{\eta_{t} B_{t}}\right)^{\frac{1}{\phi_{t}}}\right]^{\gamma_{t}-1}}{z_{t} A_{t} k_{t}^{\alpha_{t}}\left[h_{t}-\left(\frac{h_{t+1}}{\eta_{t} B_{t}}\right)^{\frac{1}{\phi_{t}}}\right]^{\gamma_{t}}-k_{t+1}}\right\}=0 .}
\end{gathered}
$$

By using the optimal policy for physical capital (A2) for both terms $k_{t}$ and $k_{t+1}$, the last equation simplifies into

$$
\frac{\frac{\gamma_{t-1}}{\phi_{t-1}}\left(\frac{h_{t}}{\eta_{t-1} B_{t-1}}\right)^{\frac{1}{\phi_{t-1}}}}{\left[h_{t-1}-\left(\frac{h_{t}}{\eta_{t-1} B_{t-1}}\right)^{\frac{1}{\phi_{t-1}}} h_{t}\right.}=\beta \mathbb{E}_{t-1}\left\{\frac{\gamma_{t}}{\left[h_{t}-\left(\frac{h_{t+1}}{\eta_{t} B_{t}}\right)^{\frac{1}{\phi_{t}}}\right]}\right\}
$$

From the original dynamic constraint in (2), we can recover the control variable formulation for human capital and substitute $h_{t-1}-\left[h_{t} /\left(\eta_{t-1} B_{t-1}\right)\right]^{1 / \phi_{t-1}}$ with $h_{t-1} u_{t-1}$ and $h_{t}-\left[h_{t+1} /\left(\eta_{t} B_{t}\right)\right]^{1 / \phi_{t}}$ with $h_{t} u_{t}$, while also noting that $\left[h_{t} /\left(\eta_{t-1} B_{t-1}\right)\right]^{1 / \phi_{t-1}}$ $=\left(1-u_{t-1}\right) h_{t-1}$, thus obtaining

$$
\frac{\gamma_{t-1}\left(1-u_{t-1}\right) h_{t-1}}{\phi_{t-1} h_{t-1} u_{t-1} h_{t}}=\beta \mathbb{E}_{t-1}\left(\frac{\gamma_{t}}{h_{t} u_{t}}\right)
$$

which, again after pulling $h_{t}$ out of the expectation from the RHS as it is a deterministic choice taken at time $t-1$ with all the information available at that moment (while $u_{t}$, representing a decision to be taken at time $t$, is still unknown at time $t-1$ ), and simplifying terms, becomes

$$
\frac{\gamma_{t-1}\left(1-u_{t-1}\right)}{\phi_{t-1} u_{t-1}}=\beta \mathbb{E}_{t-1}\left(\frac{\gamma_{t}}{u_{t}}\right)
$$


Under the i.i.d. assumption, we can safely assume that the expectation on the RHS is constant, say $\mathbb{E}_{t-1}\left(\gamma_{t} / u_{t}\right)$ $=\mathbb{E}(\gamma / u) \equiv C$ (since the realization $\gamma_{t}$ is associated with a unique configuration $\left(z_{t}, \eta_{t}, \alpha_{t}, \gamma_{t}, \phi_{t}, A_{t}, B_{t}\right)$ of shocks, it is reasonable to assume that the optimal choice for $u_{t}$ must be the same whenever such a configuration is realized) and then rearrange the last equation as

$$
\frac{\gamma_{t-1}}{u_{t-1}}=\gamma_{t-1}+\beta C \phi_{t-1}
$$

which, taking expectations on both terms, turns into

$$
\mathbb{E}\left(\frac{\gamma_{t-1}}{u_{t-1}}\right)=\mathbb{E}\left(\frac{\gamma}{u}\right)=C=\mathbb{E}(\gamma)+\beta C \mathbb{E}(\phi),
$$

where under the i.i.d. assumption $\mathbb{E}(\gamma)=\sum_{i=1}^{m} p_{i} \gamma_{i}$ and $\mathbb{E}(\phi)=\sum_{i=1}^{m} p_{i} \phi_{i}$ are constants, yielding the expected ratio

$$
\mathbb{E}\left(\frac{\gamma}{u}\right)=C=\frac{\mathbb{E}(\gamma)}{1-\beta \mathbb{E}(\phi)} .
$$

Using the last expression for $\mathbb{E}_{t-1}\left(\gamma_{t} / u_{t}\right)$ in (A3), the optimal fraction of human capital to be employed in the final good production as in (4) is immediately obtained.

Since the partial derivatives of the instantaneous utility along the optimal path $\left(k_{t}^{*}, h_{t}^{*}\right)$ defined by (5) are

$$
\frac{\partial}{\partial k_{t}} u=\frac{\beta \mathbb{E}(\alpha)}{[1-\beta \mathbb{E}(\alpha)] k_{t}^{*}} \quad \text { and } \quad \frac{\partial}{\partial k_{t}} u=\frac{\beta \mathbb{E}(\gamma)}{[1-\beta \mathbb{E}(\phi)] h_{t}^{*}},
$$

the transversality condition

$$
\lim _{t \rightarrow \infty} \beta^{t} \mathbb{E}_{0}\left\{\frac{\beta \mathbb{E}(\alpha)}{[1-\beta \mathbb{E}(\alpha)] k_{t}^{*}} k_{t}^{*}+\frac{\beta \mathbb{E}(\gamma)}{[1-\beta \mathbb{E}(\phi)] h_{t}^{*}} h_{t}^{*}\right\}=0
$$

is satisfied and the proof is complete.

Acemoglu, D. and Restrepo, P., Artificial Intelligence, Automation and Work (MIT Department of Economics Working Paper Series, 2018), Paper No. 18-01.

Barnsley, M., Fractals Everywhere, 2nd ed. (Academic Press, San Diego, CA, 1993).

Barnsley, M., Ervin, V., Hardin, D., and Lancaster, J., "Solution of an inverse problem for fractals and other sets," Proc. Natl. Acad. Sci. U. S. A. 83, 1975-1977 (1986).

Barro, R. J. and Sala-i-Martin, X., Economic Growth (MIT Press, Cambridge, MA, 2004).

Benhabib, J. and Perli, R., "Uniqueness and indeterminacy: Transitional dynamics in a model of endogenous growth," J. Econ. Theory 63, 113-142 (1994).
Brock, W. A. and Mirman, L. J., "Optimal economic growth and uncertainty: The discounted case," J. Econ. Theory 4, 479-513 (1972).

Hutchinson, J., "Fractals and self-similarity," Indiana Univ. J. Math. 30, 713-747 (1981).

IPCC, "Climate change 2007: Synthesis report," Intergovernmental Panel on Climate Change, 2007.

Korinek, A. and Stiglitz, J. E., "Artificial intelligence and its implications for income distribution and unemployment," NBER Working Paper No. 24174, 2017.

Kunze, H., La Torre, D., Mendivil, F., and Vrscay, E. R., Fractal-based Methods in Analysis (Springer, New York, 2012).

La Torre, D., Marsiglio, S., and Privileggi, F., "Fractals and self-similarity in economics: The case of a two-sector growth model," Image Anal. Stereol. 30, 143-151 (2011).

La Torre, D., Marsiglio, S., Mendivil, F., and Privileggi F., "Self-similar measures in multi-sector endogenous growth models," Chaos, Solitons Fractals 79, 40-56 (2015).

La Torre, D., Marsiglio, S., Mendivil, F., and Privileggi F., "Fractal attractors and singular invariant measures in two-sector growth models with random factor shares," Commun. Nonlinear Sci. Numer. Simul. 58, 185-201 (2018).

Lucas, R. E., "On the mechanics of economic development," J. Monetary Econ. 22, 3-42 (1988)

Marsiglio, S., "Stochastic shocks in a two-sector solow model," Int. J. Math. Modell. Numer. Optim. 3, 313-318 (2012).

Marsiglio, S., Ansuategi, A., and Gallastegui, M. C., "The environmental kuznets curve and the structural change hypothesis," Environ. Resource Econ. 63, 265-288 (2016).

Marsiglio, S. and La Torre, D., "Economic growth and abatement activities in a stochastic environment: a multi-objective approach," Ann. Oper. Res. (published online, 2016).

Mitra, T., Montrucchio, L., and Privileggi, F., "The nature of the steady state in models of optimal growth under uncertainty," Econ. Theory 23, 39-71 (2003).

Mitra, T. and Privileggi, F., "Cantor type invariant distributions in the theory of optimal growth under uncertainty," J. Differ. Eq. Appl. 10, 489-500 (2004).

Mitra, T. and Privileggi, F., "Cantor type attractors in stochastic growth models," Chaos, Solitons Fractals 29, 626-637 (2006).

Mitra, T. and Privileggi, F., "On lipschitz continuity of the iterated function system in a stochastic optimal growth model," J. Math. Econ. 45, 185-198 (2009).

Montrucchio, L. and Privileggi, F., "Fractal steady states in stochastic optimal control models," Ann. Oper. Res. 88, 183-197 (1999).

Nordhaus, W., "Integrated economic and climate modeling," in Handbook of Computable General Equilibrium Modeling, edited by Dixon, P. B. and Jorgenson, D. (Elsevier, Amsterdam, 2013), Vol. 1, pp. 1069-1131.

Privileggi, F. and Marsiglio, S., "Environmental shocks and sustainability in a basic economy-environment model," Int. J. Appl. Nonlinear Sci. 1, 67-75 (2013).

Rebelo, S., "Long-run policy analysis and long-run growth," J. Political Econ. 99, 500-521 (1991).

Soretz, S., "Stochastic pollution and environmental care in an endogenous growth model," Manchester School 71, 448-469 (2003).

Vrscay, E. R., "Moment and collage methods for the inverse problem of fractal construction with iterated function systems," in Fractals in the Fundamental and Applied Sciences, edited by Peitgen, H. O., Henriques, J. M., and Penedo, L. F. (North-Holland, Amsterdam, 1991), pp. $443-461$. 